\title{
The Health Impacts of Climate Change: Getting Started on a New Theme
}

\author{
Kristie L. Ebi, PhD, MPH; ${ }^{1}$ Madeleen Helmer; ${ }^{2}$ Jari Vainio, $\mathrm{PhD}^{3}$
}

1. President, ESS, LLC, Independent Consultant on Climate Change Issues

2. Head, Red Cross/Red Crescent Climate Centre, The Hague, the Netherlands

3. Senior Health officer, Public Health in Emergencies Unit in the International Federation of Red Cross and Red Crescent Societies, Geneva, Switzerland.

\author{
Correspondence: \\ Madeleen Helmer \\ Head Red Cross/Red Crescent Climate \\ Centre \\ PO Box 28120, $2502 \mathrm{KC}$ \\ The Hague, the Netherlands \\ E-mail: climatecentre@redcross.nl
}

This targeted agenda program was sponsored and supported by Red Cross/Red Crescent Climate Centre, The Hague, The Netherlands

Keywords: climate change; extreme weather events; health impacts

\author{
Abbreviations: \\ IFRC = International Federation of Red \\ Cross and Red Crescent Societies \\ IPCC = Intergovernmental Panel on Climate \\ Change \\ $\mathrm{RC} / \mathrm{RC}=$ Red Cross/Red Crescent \\ TAP $=$ Targeted Agenda Program \\ 15WCDEM $=15^{\text {th }}$ World Congress on \\ Disaster and Emergency Medicine \\ WHO $=$ World Health Organization
}

Web publication: 27 August 2008

\begin{abstract}
Climate change is widely acknowledged as a key global challenge for the 21 st century, and is projected to significantly affect population health and human well-being. All of the climate change-related changes in weather patterns will affect human health, from boosting mental well-being to mortality from largescale disasters. Human health can be affected both directly and indirectly.

For various reasons, the health sector has been slow in responding to the projected health impacts of climate change. To effectively prepare for and cope with climate change impacts, public health must move from a focus on surveillance and response to a greater emphasis on prediction and prevention.

The targeted agenda program dialogue identified three priorities for climate change related health actions: heat waves, vector-borne diseases; and malnutrition.
\end{abstract}

Ebi KL, Helmer M, Vainio J: The health impacts of climate change; Getting started on a new theme. Prehospital Disast Med 2008;23(4):s60-s64.

\section{Introduction}

In 2005, anticipating the 15th World Congress on Disaster and Emergency Medicine in Amsterdam from 13-15 May 2007 (15WCDEM), the Red Cross/Red Crescent (RC/RC) Climate Centre was invited to organize a targeted agenda program (TAP) on the health effects of climate change. At that moment, the issue of climate change received limited attention and the $\mathrm{RC} / \mathrm{RC}$ Climate Centre anticipated that the TAP process would generate further awareness on the issue and would support the creation of a network of experts on the issue. By the time the Opening Ceremony of 15WCDEM took place, climate change had become a key global challenge for the 21st century, and is projected to significantly affect population health and human well-being. ${ }^{1}$

Climate change will be characterized by increased temperatures, changes in the hydrologic cycle, sea level rise, and increased climate variability, particularly more frequent and intense heat waves, droughts, and floods. ${ }^{2}$ All of these changes will affect human health from boosting mental well-being (many people would like the weather to become warmer) to mortality from large-scale disasters. Human health will be affected both directly and indirectly.

This article first briefly summarizes global assessments of future disease burdens possibly attributable to climate change, and summarizes the potential climate change-related health impacts of heat waves, floods, droughts, and windstorms. In addition, the indirect health impacts of climate change on vector-borne diseases are addressed. Impacts on food- and water-borne diseases, and on diseases related to air pollutants and aeroallergens are not dealt with in this summary.

The first part of this article was prepared prior to the 15WCDEM as a starting document for discussion between the TAP members. It provided contextual information on the issue. The second part of the article summarizes the TAP discussions and results during the 15WCDEM. 


\section{Methods}

For the RC/RC Climate Centre, the invitation to participate in the 15WCDEM came at the ideal time because the main focus of the Centre so far had been on climate change and disaster risk reduction. Though health effects of climate change had arisen at different times in the last five years, in particular in relation to the European heat wave of 2003, the subject had not received the attention needed. The TAP provided a good opportunity for change.

In cooperation with Kristie L. Ebi, Intergovernmental Panel on Climate Change (IPCC) author on Health, and the International Federation of Red Cross and Red Crescent Societies (IFRC), a process was initiated. The Health and Care Department of the IFRC appointed a focal point for climate change, Mr. Jari Vainio. Two experts, Mrs. Franzisca Matthies of the World Health Organisation (WHO) and Professor Glenn McGregor of King's College in London joined the TAP-meeting during the Congress in May.

The TAP dialogue had three aims:

1. Identify the current knowledge and research initiatives and networks regarding the impacts of climate change on health, particularly in the context of extreme weather events and disasters;

2. Identify gaps in existing knowledge and research that should be bridged to strengthen the engagement of the health sector; and

3. Define ways forward to increase the understanding of climate change risks for the health sector and to strengthen the engagement of the health sector to address these risks.

\section{Part 1: The Context}

\section{Direct Health Impacts}

Directly, extreme weather events (including heat waves, floods, droughts, and windstorms) annually affect millions of people and cause billions of dollars of damage. In Europe, Canada, and the United States, in 2003, floods and storms resulted in 101 people dead or missing, and caused US $\$ 9.73$ billion in insured damages. ${ }^{3}$ More than 35,000 excess deaths were attributed to the extended heat wave in Europe the same year. The health impacts of extreme events in developing countries are substantially larger. There is scientific consensus that the frequency and intensity of extreme weather events likely will increase during the coming decades as a consequence of climate change ${ }^{4,5}$ the associated health impacts also could increase.

Heat Waves-Heat waves affect human health via heat stress, heatstroke, and death, ${ }^{6}$ as well as exacerbations of underlying conditions that can increase mortality. ${ }^{7}$ The frequency and intensity of heat events ${ }^{5}$ and heat-related deaths are projected to increase with climate change. 8,9 However, health projections have not taken into account very large changes in heat wave intensity, such as occurred in 2003 in Europe; nor have they estimated impacts in developing countries where increasing temperatures could affect human and agricultural productivity.

Floods, Droughts, and Windstorms-The health and social burden of extreme weather events can be quite large, caus- ing loss of life and livelihood, infrastructure damage, population displacement, and economic disruption. The impact of an extreme event is determined by the physical characteristics of the event, attributes of the location affected, and interactions of these with human actions and social, economic, institutional, and other systems. Extreme events can have local and regional effects. Climate change is projected to increase the intensity and frequency of extreme weather events in many regions. ${ }^{10}$

The adverse health consequences of flooding and windstorms can be complex and far-reaching. Adverse health impacts include the physical health effects experienced during the event or clean-up process, or from effects brought about by damage to infrastructure, including population displacement. The physical effects largely manifest themselves immediately, within weeks or months following the event, and can be direct and immediate (such as injuries) or indirect and delayed (such as water and food shortages and increased rates of vector-borne diseases and diarrheal diseases due to water contamination or reduced water availability). Extreme weather events also are associated with mental health effects resulting from the experience of the event or from the recovery process. These psychological effects tend to be long lasting and can be worse than the direct physical effects. ${ }^{11}$ The effects of drought on health include malnutrition (protein-energy malnutrition and/or micronutrient deficiencies), infectious and diarrheal diseases, and respiratory diseases. ${ }^{12}$ In addition, malnutrition increases the risk of dying from an infectious disease. The loss of livelihoods due to drought is a major trigger for population movements, which can cause additional disease burdens.

Parry et al projected that the world will have sufficient food to feed everyone up to the end of the 21st century, under all climate change scenarios; ${ }^{13}$ however, this assumed that people in low-income countries, where climate change impacts are predominantly negative, would have access to food produced in the more developed countries

Although data are limited, malnutrition associated with drought and flooding may be one of the most important consequences of climate change due to the large number of people that may be affected. One study projected that climate change could increase the percentage of the Malian population at risk for hunger from $34 \%$ to $64-72 \%$ by the 2050 s, although this could be reduced by implementation of a range of adaptive strategies.

\section{Indirect Health Impacts}

Indirectly, climate can affect health through alterations in the geographic range and intensity of transmission of vector-, tick-, and rodent-borne diseases and food- and waterborne diseases, and changes in the prevalence of diseases associated with air pollutants and acroallergens. These health outcomes include several major causes of ill health, including undernutrition, which affects $17 \%$ of the world's population in developing countries; diarrheal diseases and other conditions due to unsafe drinking water, and the lack of basic sanitation, which cause two million deaths annually, mostly in young children; ${ }^{14}$ and malaria, which causes more than one million childhood deaths annually. Climate 


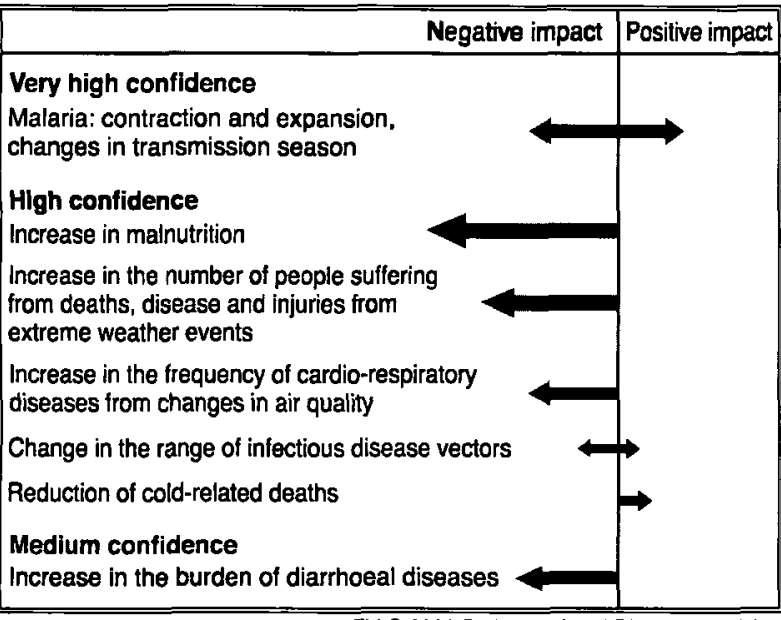

Ebi $\odot 2008$ Prehospital and Disaster Medicine

Figure 1-Summary of the relative direction, magnitude, and certainty of climate change-related health impacts Source: Confalonieri U, Menne B, et al $2007^{17}$

change could alter or disrupt natural systems, making it possible for diseases to spread or emerge in areas where they had been limited or had not existed, or for diseases to disappear by making areas less hospitable to the vector or the pathogen.

Vector-Borne Diseases-Climate is a primary determinant of whether a particular location has environmental conditions suitable for the transmission of several vector-, rodent-, and tick-borne diseases, like malaria and dengue. A change in temperature may hinder or enhance vector and parasite development and survival-thus lengthening or shortening the season during which vectors and parasites can survive. Small changes in temperature or precipitation may cause previously inhospitable altitudes or ecosystems to become conducive to disease transmission or cause currently hospitable conditions to become inhospitable. While climate is an important driver of malaria and other diseases, it is not the only one-the non-climatic socioeconomic and biological drivers include drug and pesticide resistance, deterioration of health care, deterioration of public health infrastructure including vector control efforts, demographic change, and changes in land use.

Given that malaria is a complex disease to model and that current models need improvement, models suggest that, in Africa, climate change may be associated with both expansions and contractions of the geographic area suitable for transmission of stable Plasmodium falciparum malaria, with expansion projected to be larger than contraction. ${ }^{15}$ These projections are consistent with experiences with malaria control officers in the field. Some projections suggest that the season of transmission may be extended, which may be as important for disease burdens (and vector control programs) as geographical expansion.

Climate also can impact population health through consequences of climate-induced economic dislocation and environmental decline. Climate change is projected to alter the geographical range and incidence of these and other cli- mate-sensitive health outcomes. ${ }^{16}$ The relative direction, magnitude, and certainty of climate change-related health impacts are summarized in Figure 1.

The cause-and-effect chain from climate change to changing patterns of health determinants and outcomes often is complex and includes factors such as wealth, distribution of income, status of the public health infrastructure, provision of medical care, and access to adequate nutrition, safe water, and sanitation. Therefore, the severity of future impacts will be determined by changes in climate as well as by concurrent changes in non-climatic factors and by the adaptation measures implemented to reduce negative impacts (Figure 2).

As shown in Figure 3, while populations in all countries will be exposed to increased health risks, the risks will be much greater in low-income countries because the current burden of climate-sensitive health outcomes is high, and because public health systems that could substantially reduce health risks tend to be relatively weak. Although low-income countries are likely to suffer a larger burden of health impacts, high-income countries also are likely to experience increased risks, as demonstrated by recent events. Failing to invest in adaptation may leave populations poorly prepared, and may increase the probability of severe adverse consequences.

Increasing Health Sector Engagement in Climate Change Although climate change-related health risks are clear, there has been limited engagement of the health sector in modifying current and implementing new interventions to manage the risks. This is due partly to limited understanding of the immediacy of the climate change risks and partly due to significant, competing demands. Increasing awareness of the following will help strengthen engagement of the health sector:

1. Anthropogenic climate change is occurring, including both changes in the mean and variance of weather variables;

2. Health impacts attributable to climate change are occurring;

3. Inertia in the climate system means that change will continue for decades after successful control of greenhouse gas emissions; and

4. The extent of health impacts will depend on the ability of the public health sector to modify current and implement new effective and timely adaptation measures.

To effectively prepare for and cope with climate change impacts, public health must move from a focus on surveillance and response to a greater emphasis on prediction and prevention.

Early warning systems for floods and heat waves have been shown to be effective in reducing mortality. Health sector engagement is needed for developing early warning systems of extreme weather events to both reduce current vulnerability to extreme events and increase future coping capacity. The principal components of a public health early warning system include meteorological forecasts, models to predict health outcomes, an effective response plan, and a monitoring and evaluation plan, all set within a disaster 


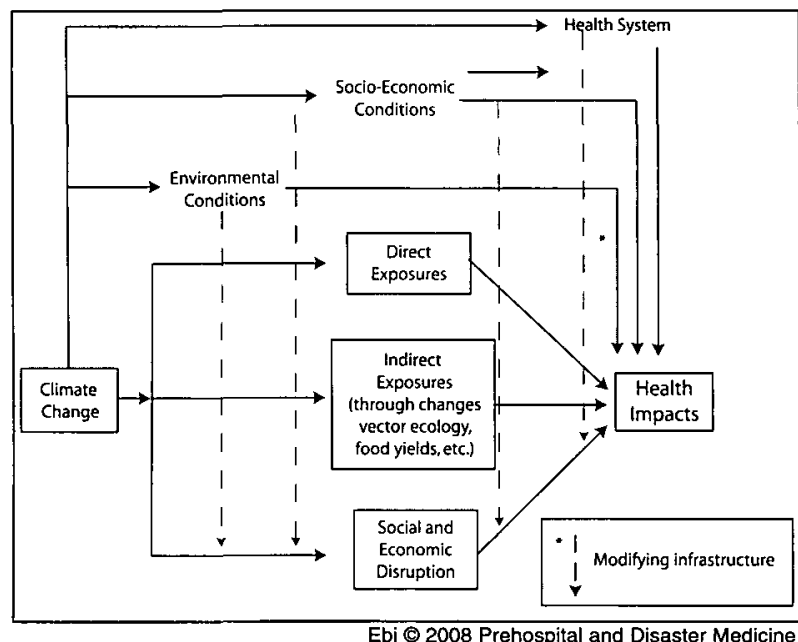

Figure 2-Pathways by which climate change affects population health and concurrent direct-acting and modifying influences

Adapted from: Confalonieri U, Menne B, et al $2007^{17}$

management strategy. ${ }^{17}$ Because early warnings alone are not sufficient to guarantee that necessary actions will be taken, prevention programs must be designed with a better understanding of sub-populations at risk and the information necessary for effective response to warnings. Monitoring and evaluation of both the system and individual interventions must be built into system design to ensure the efficient and effective use of resources.

\section{Part 2: Results of the TAP Sessions and Discussions}

During the TAP sessions, it became clear that there are a growing number of knowledge and research initiatives and networks on climate change and health, including:

1. The Red Cross/Red Crescent network on climate change and health, including the Preparedness for Climate Change program;

2. The World Health Organization (WHO)/United Nations Development Programme Global Environment Facility (UNDP/GEF) project, "Piloting Climate Change Adaptation to Protect Human Health";

3. The WHO workshops on climate change and health (10 workshops will have been conducted by the end of 2007);

4. Projects coordinated by the WHO Europe Regional Office, including EUROHEAT, climate Change and Adaptation Strategies for Human Health in Europe, and others;

5. Second National Communications under the United Nations Framework Convention on Climate Change (UNFCCC) (for low- and middle-income countries);

6. The World Meteorological Organization (WMO) Commission developing guidance notes on heat wave and health early warning systems; and

7. Research conducted under the global change SysTem for Analysis, Research, and Training project (START), "Assessments of Impacts and Adaptation to Climate Change".

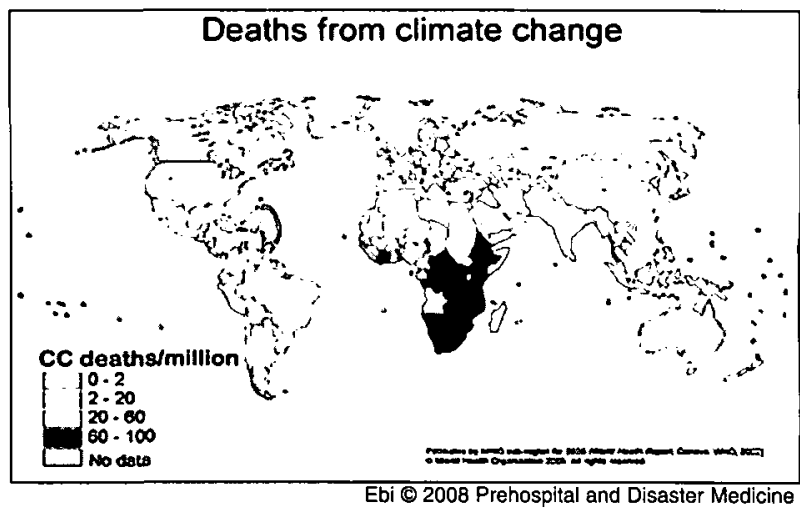

Figure 2-Deaths from climate change, 2000 Source: Patz et al 2006.

In addition, research agendas have been articulated by several groups, including the research agenda of the Human Health Chapter of the $4^{\text {th }}$ Assessment Report of the Intergovernmental Panel on Climate Change. Although significant knowledge gaps exist, there is sufficient information on the health impacts of climate variability and change for adaptation activities to be initiated immediately. A key activity is to ensure that consideration of the risks of and responses to climate change are included in all relevant strategies, policies, and measures.

The participants in the TAP dialogue concluded that there are three priorities for early action:

1. Heat waves, with a particular focus on Europe and India. These regions were emphasized because both have experienced recent heat waves and resulted in significant numbers of deaths, and because both regions have active research and/or public health intervention programs addressing this issue;

2. Vector-borne diseases, particularly malaria in Africa, currently cause large health burdens, with climate change likely to place considerable pressure on current vector control activities. Epidemic malaria can lead to high morbidity and mortality, creating crises to which the Red Cross/Red Crescent and others will have to respond; and

3. Malnutrition linked to drought is projected to increase with climate change. Increasing temperatures with climate change increase evapotranspiration, which decreases soil moisture in areas with limited precipitation. One of the conclusions of the IPCC $4^{\text {th }}$ Assessment Report is that increasing temperatures will put rain-fed agriculture in Sub-Saharan Africa at risk; this could lead to increasing malnutrition in an already struggling region.

Another issue discussed is that current and projected climate change will interact with other large-scale risks, including violence, the threat of disease pandemics (presently avian influenza), and migration. The options for coping with these issues should not be considered in isolation; otherwise choices could be made that decreased vulnerability to one risk while increasing vulnerability to another. One tool for approaching these large, complex, and interacting issues is the development of scenarios that can be used for education and training and for planning purposes. 


\section{Conclusions}

The TAP on climate change and health was successful in that it created an excellent opportunity to discuss, and for many for the first time, the health impacts of climate change, within the World Association of Disaster and Emergency Medicine (WADEM). Yet, because the subject was so new, the TAP may, in hindsight, not have been the most effective form to address this issue for the WADEMmembers. The TAP, in order to have a concrete result, was already too prepared to invite WADEM participants during the Congress to have a meaningful input on this new subject. Also, the TAP was hindered by the fact that the

References

1. Intergovernmental Panel on Climate Change: Climate Change 2007, 4th Assessment Report, Geneva 2007.

2. Intergovernmental Panel on Climate Change: Climate Change 2007, The Pbysical Science Base, Geneva 2007

3. Swiss Re: Natural catastrophes and man-made disasters in 2003, Sigma ins research $1 / 2004$

4. Easterling D, Meehl GA, Parmesan C, et al: Climate extremes: Observations, modeling and impacts. Science 2000;289(5485):2068-2074.

5. Meehl GA, Tebaldi $\mathrm{C}$ : More intense, more frequent, and longer lasting heat waves in the 21st century, Science 2004;305(5686):994-997.

6. Kilbourne E: Heat Waves and Hot Environments. In: The Public Health Consequences of Disasters. Oxford University Press, 1997.

7. Kovats RS, Koppe C: Heat Waves: Past and Future Impacts. In: Integration of Public Health with Adaptation to Climate Cbange: Lessons Learned and New Directions. Taylor \& Francis, London UK, pp 136-160.

8. Desai S: Heat stress and mortality in Lisbon Part II. An assessment of the potential impacts of climate change. Int Journal for Biometeorology 2003;48(1):37-44.

9. Hayhoe K, Cayan D, Field CB, et al: Emission pathways, climate change and impacts on California. Proc Natl Acad Sci USA 2004;101(34):12422-12427. organizers were not active members of the WADEM. Therefore, Synergy with ongoing processes within the WADEM was not created. For the organizers of the TAP, it was a meaningful process because it led to strengthened cooperation between the participants and a more focused approach on climate change and health within the IFRC.

\section{TAP Members}

Kristie L. Ebi, Intergovernmental Panel on Climate Change (IPCC), Jari Vainio, IFRC focal point for climate change, Franzisca Matthies, World Health Organization Glenn McGregor, King's College in London, Madeleen Helmer, Head RC/RC Climate Centre.
10. Ahern M, Kovats RS, Wilkinson P, et al: Global health impacts of floods: Epidemiologic evidence. Epidemiologic Reviews 2005;27(1):36-46.

11. Menne B; Bertollini R: The health impacts of desertification and drought The Newsletter of the Convention to Combat Desertification, 2000

12. Parry ML, Rosenzweigb C, Iglesias A, et al: Effects of climate change on global food production under SRES emissions and socio-economic scenarios. Global Environmental Change 2004;14(1):53-67.

13. Kosek M, Bern C, Guerrant RL: The global burden of diarrhoeal disease, as estimated from studies published between 1992 and 2000. Bull World Healtb Organ 2003;81(3):197-204.

14. F. Tanser, Sharp B, le Sueur D: Potential effect of climate change on malaria transmission in Africa. Lancet 2003;362(9398):1792-1798.

15. Intergovernmental Panel on Climate Change: Climate Change 2007, Impacts, Vulnerability and Adaptation, Geneva 2007

16. Ebi K, Schmier J: A stitch in time: Improving public health early warning systems for extreme weather events. Epidemiological Reviews 2005;27(1): 115-121.

17. Confalonieri U, Menne B, et at: Human Health. In Parry M, Canziani O, Palutikof $\mathrm{P}$, et al: Climate Change 2007: Impacts, Adaption and Vulnerability. Contribution of Working Group II to the Fourth Assessment Report of the Intergovernmental Panel on Climate Cbange. Cambridge: Cambridge University Press, 2007. 